\title{
Causality Inversion in the Reproduction of Roughness
}

\author{
Michaël Wiertlewski ${ }^{1,2}$, José Lozada ${ }^{1}$, \\ Edwige Pissaloux ${ }^{2}$, and Vincent Hayward ${ }^{2}$ \\ 1 CEA, LIST, Sensory and Ambient Interfaces Laboratory, 18 route du Panorama, BP6, 92295 \\ Fontenay-Aux-Roses, France \\ \{michael.wiertlewski, jose.lozada\}@cea.fr \\ 2 UPMC Univ Paris 6, Institut des Systèmes Intelligents et de Robotique, 75005, Paris, France \\ \{pissaloux, hayward\}@isir.upme.fr
}

\begin{abstract}
When a finger scans a non-smooth surface, a sensation of roughness is experienced. A similar sensation is felt when a finger is in contact with a mobile surface vibrating in the tangential direction. Since an actual finger-surface interaction results in a varying friction force, how can a measured friction force can be converted into skin relative displacement. With a bidirectional apparatus that can measure this force and transform it into displacement with unambiguous causality, such mapping could be experimentally established. A pilot study showed that a subjectively equivalent sensation of roughness can be achieved betweem a fixed real surface and a vibrated mobile surface.
\end{abstract}

Key words: Roughness simulation; Haptic devices; Virtual reality.

\section{Introduction}

Roughness is an important attribute of things we touch [1]. Concomitantly, there is a need for ever increasingly realistic virtual environments that can reproduce the various attributes of objects, including their roughness. To date, the approaches used to simulate roughness include the use of force feedback devices to replicate the microgeometry of surfaces, directly, or by reproducing its effects; see [2] for an extensive survey. Other approaches modulate the friction force that arises when a finger slips on an active surface. To this end, electrostatic fields [3], surface acoustic waves [4], or the squeeze film effect [5]6], can be employed.

Finger-Surface interaction. The steady slip of a finger on a surface induces a frictional force. If the surface in question deviates from smoothness, then the interaction force varies over time as a result of a complex interaction taking place between the finger and the surface. Microscopically, the variation is the consequence of the space-andtime-varying traction distribution (i.e. tangential force per unit of contact surface) at the interface between the finger and the surface. The traction distribution depends on the relative geometries of these two bodies, on the materials they are made of, and on the possible presence of fluids and foreign bodies.

In spite of this complexity, integration of traction over the (unknown) contact surface results in a net force that can be measured. It is known that the variations of this

A.M.L. Kappers et al. (Eds.): EuroHaptics 2010, Part II, LNCS 6192, pp. 17-24 2010.

(c) Springer-Verlag Berlin Heidelberg 2010 
force correlate strongly with a sensation of roughness [7]8]. While there is much debate regarding the manner in which the nervous system mediates the sensation of roughness peripherally and centrally, there is evidence that a variety of mechanisms are at play. Because of the multiplicity of these mechanisms, diverse stimulation methods can contribute to elicit roughness, see [9] among others.

Present Study. The present paper explores the possibility of stimulating the cutaneous system in order to create roughness sensations through the simplest method possible: that of vibrating tangentially a smooth surface in non-slipping contact with the finger, as the finger undergoes net motion. Yet, when it comes to design a display based on this idea, this simple approach poses a basic question which must be clearly answered: During tactile exploration, does the finger-surface interaction force "cause" the finger to deform or does the deformation "cause" the interaction force? Visual or auditory displays, by-and-large, radiate the same energy regardless of how they are looked at or listened to, so the causality is clear, but for haptic displays the causality question cannot be answered so easily, see [10] for elementary notions. The same question can be rephrased as follows: Should the measurement and the simulation be based on the skin displacement or on the force applied to it?

Bidirectional apparatus. To study this question, we build an apparatus that unambiguously establishes a causal relationship between the measurement and the stimulation by operating both as a sensor and as an actuator. In these two cases, the device was engineered to be very stiff, that is, five orders of magnitude stiffer than a fingertip. This way, when used as a sensor, the interaction force is known regardless of the finger movements and deformations; when used as an actuator, displacement is specified independently from the interaction force. To complete the symmetry, during recording operations, the sensor is fixed with respect to the ground and the finger slips on a rough surface. During restitution, the actuator is mounted on a slider and remains fixed relatively to the scanning finger touching a flat surface. In both modes, the device operates with a bandwidth spanning from 20 to $600 \mathrm{~Hz}$, and has a maximum displacement of $0.2 \mathrm{~mm}$ in actuator mode, thereby covering the range useful for conveying roughness.

Main result. We performed a preliminary psychophysical experiment aimed at finding the subjective equivalence of roughness elicited by a rapidly varying measured force or by an imposed displacement, hence realizing a causality inversion between the measurement and the display. This approach is in contrast with the one employed with conventional haptic devices where a force is measured, or computed, and then specified with impedance devices; or where a displacement is computed and then specified with admittance devices. It was found that, indeed, such subjective equivalence of roughness could be established.

\section{Apparatus}

Referring to Figs. 19 and 3 , the apparatus comprises a rigid plate, A supported at one end by a low stiffness blade, $B$, and connected to a multilayer piezoelectric circular bender (CMBR07, Noliac Group A/s, Kvistgaard, Denmark), C, at the other. As a sensor, a 
textured surface is glued to the plate and during scanning the interaction force is measured within a very large dynamic range. As an actuator, the assembly is mounted on a linear guide and the smooth plate is vibrated tangentially.

\subsection{Sensor Operation}

The piezoelectric bender converts tangential forces due to the interaction with the finger into electric charges. These charges are transformed into voltage by an instrumentation amplifier (LT 1789, Linear Technology Corp., Milpitas, CA, USA) as shown in Fig. 1b. The signal is then digitized by a 16-bit data acquisition board (PCI-6229, National Instruments Corp., Austin, TX, USA). The piezoelectric transducer acts like a generator $V_{p}$ in series with a capacitor $C_{p}$ and a charge resistance $R_{s}$. The RC circuit corresponds to a $20 \mathrm{~Hz}$ high-pass filter. Such charge-based force sensor is capable of a very high dynamic range response unachievable with conventional strain-gauge-based force sensors.
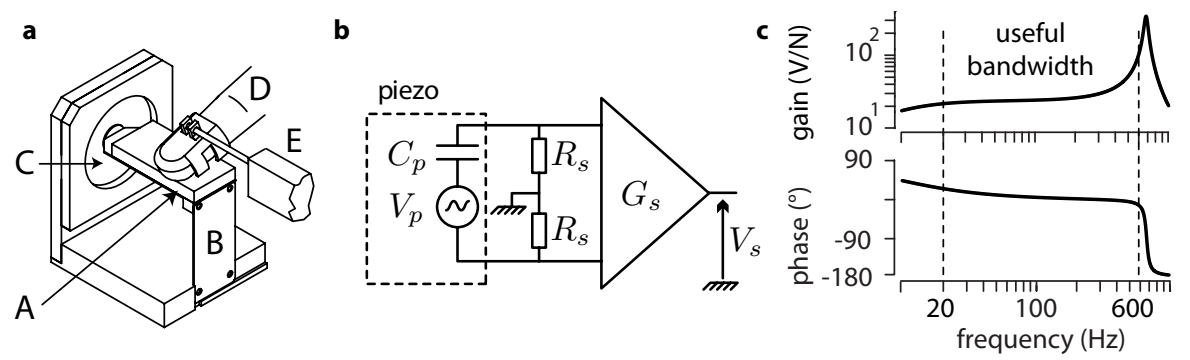

Fig. 1. Sensor operation. a: Setup. The finger position, measured by E, and the interaction force, measured by $C$ are recorded when the finger, D, slips on the surface, A. b: Signal conditioning. c: Frequency response of the sensor.

The position of the finger, D, is measured with a linear variable transformer transducer (LVDT), E, (SX 12N060, Sensorex SA, Saint-Julien-en-Genevois, France) fastened to the fingernail. The response, Fig 1; , shows a sensitivity of $26 \mathrm{~V} / \mathrm{N}$ in the range from $20 \mathrm{~Hz}$ to $600 \mathrm{~Hz}$. The range is limited upward by the mechanical natural resonance. Output noise is lower than $20 \mu \mathrm{N} / \sqrt{\mathrm{Hz}}$ and 16-bit digital conversion provides $50 \mu \mathrm{N}$ of resolution at a $2 \mathrm{kHz}$ sampling rate. The high stiffness of the bender $\left(70 \cdot 10^{3} \mathrm{~N} / \mathrm{m}\right)$ ensures that the small deformation hypothesis is valid. Low frequency force components are measured by a conventional force sensor (Nano 17, ATI Industrial Automation, Inc., Apex, NC, USA) mounted on the load path between the assembly and a firm mechanical ground.

The interaction force components $F_{t}$ and $F_{n}$ and finger position $x(t)$ are acquired by the sensor during scanning, see Fig $2 \mathrm{~b}$. A typical measurement is seen in Fig. 2b. Notice how the tangential force $F_{t}$ rises at the beginning of the motion and then oscillate around a value. The high-pass filter preserves the variation of the tangential force occurring within a wide dynamic range that a conventional force sensor would be unable to resolve, as shown in Fig.26. The initial stick to slip transition and ensuing transients have been edited out for clarity. This diagram is representative of the rich variations of the friction force due to a finger slipping on a periodic grooved surface. 

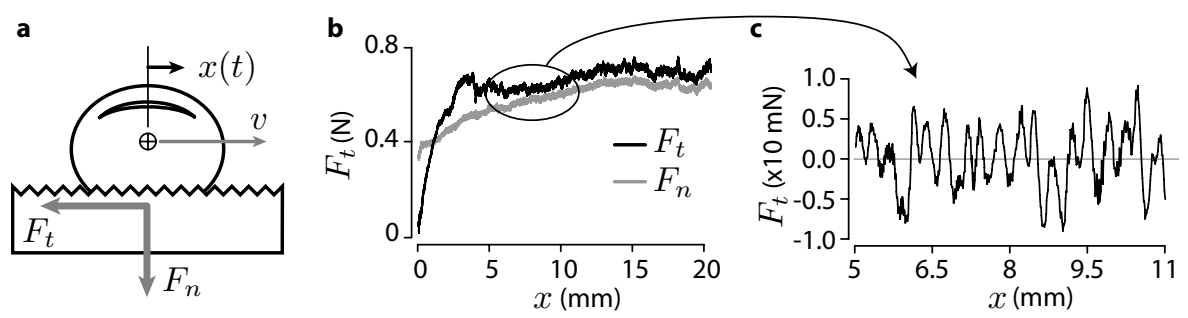

Fig. 2. Measurement a: The components $F_{n}$ and $F_{t}$ of the interaction force during scanning. $x(t)$ is measured by a LVDT. b: Typical force measurement from the conventional force sensor. c: Wide range dynamic measurement of sliding interaction measured by the piezoelectric sensor.

\subsection{Actuator Operation}

Referring to Fig. 3h, when used as an actuator, the assembly is disconnected from the grounded force sensor and placed on a slider, E. Its position is measured with a $7.5 \mu \mathrm{m}$ linear resolution using an incremental encoder, F, (Model 2400, Fritz Kübler GmbH, Villingen-Schwenningen, Germany). The participant's third phalanx rests on a cradle, $\mathrm{G}$, connected to the slider so that the fingertip rests on the active surface, A. As the finger scans to and fro, the transducer is driven by a voltage amplifier (Apex Precision Power PA86U, Cirrus Logic Inc., Austin, TX, USA) such that the skin in contact with the active surface is entrained by its oscillations without slip.
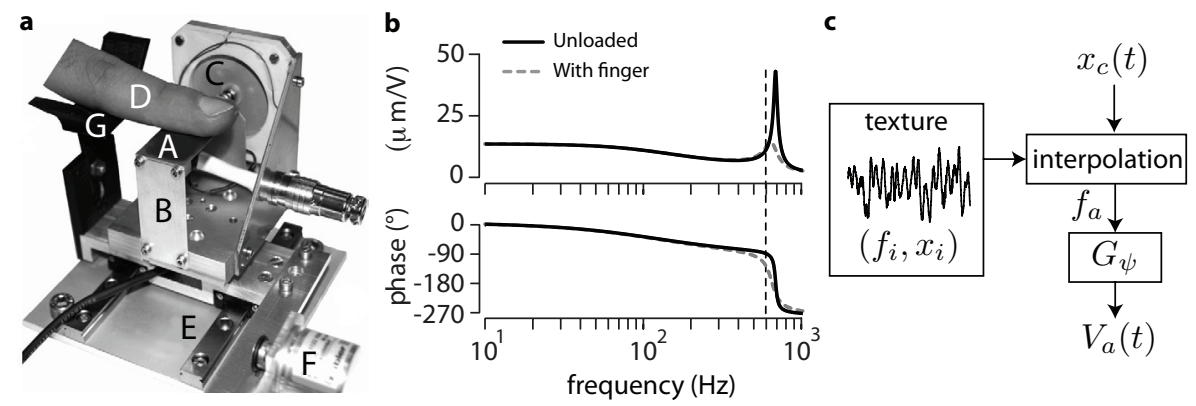

Fig. 3. Actuator operation. a: The stimulator, C, mounted on a linear stage, E, is fixed relatively to the finger, D. b: Frequency response of the none loaded actuator (black line) and with a finger pushing at $1 \mathrm{~N}$ (dash line). c: Control.

In order to ascertain performance, the output displacement was measured with a laser telemeter (LT2100, Keyence Corp., Osaka, Japan). The response, Fig. 3b, shows that the system is able to produce a displacement of $\pm 20 \mu \mathrm{m} / \mathrm{V}$ from DC to $600 \mathrm{~Hz}$, limited by the system's natural resonance. For a $5 \mathrm{~V}$ input, the actuator is able to achieve a maximum displacement of $100 \mu \mathrm{m}$.

The actuator is driven by a $2 \mathrm{kHz}$ periodic realtime thread that reads the encoder position $x_{c}(t)$, interpolates a force value $f_{a}$ from a given texture profile, multiplies it by a gain $G_{\psi}$ and refreshes the amplifier output $V_{a}(t)$, see Fig. 3 k. This control thread runs 
under the LabVIEW ${ }^{\mathrm{TM}}$ environment on an ordinary computer equipped with the digital input-output board already mentioned.

\section{Experimental Procedure for Perceptual Calibration}

Having unambiguously converted a varying interaction force into a skin displacement during the scanning of a surface by means of an apparatus designed to establish robust causal relationships not achievable with conventional haptic devices, the question now arises of the value of the conversion factor that could elicit an equivalent sensation of roughness. Furthermore, if such a factor exists, does it vary from person to person? To address these questions, a calibration procedure was carried out with six participants in order to establish the point of subjective equivalent roughness between the natural texture and its simulated version. A 2-alternative forced choice, constant stimuli procedure was employed to find the gains $\hat{G}_{\psi}$ that would elicit an equivalent sensation of roughness.

Stimuli. The standard stimulus was a triangular grooved grating of $1 \mathrm{~mm}$ spatial period with $0.1 \mathrm{~mm}$ of depth. Without relative motion, the roughness of this texture was not perceptible. The scanning force with this grating was measured using the sensor described earlier with the help of a "standard" participant. During recording, the speed $v$ and the normal force $F_{n}$ were held constant with a $10 \%$ tolerance. The signal was processed as described in Section 2.1, then normalized to $\pm 0.5 \mathrm{~V}$. The filtered signal, expressed in Newton, and its Fourier transform are shown in Fig. 4h. The comparison stimulus was provided by the stimulator described in Section 2.2 Fig. 4h illustrates the precautions that were taken so that both stimuli were presented in exactly the same conditions: (a) The participants had their proximal phalanx resting in cradles connected to sliding guides so that the fingertip rested on the grooved texture or stimulator in same manner; (b) Both surfaces were made in polycarbonate; (c) The two sliders were mechanically connected so the same inertia and the same friction was felt for the standard and the comparison stimuli. The experimental setup was hidden by a curtain to avoid visual bias. Subjects wore sound isolation headphones (model K518, AKG Acoustics, Harman International Industries) playing white noise.
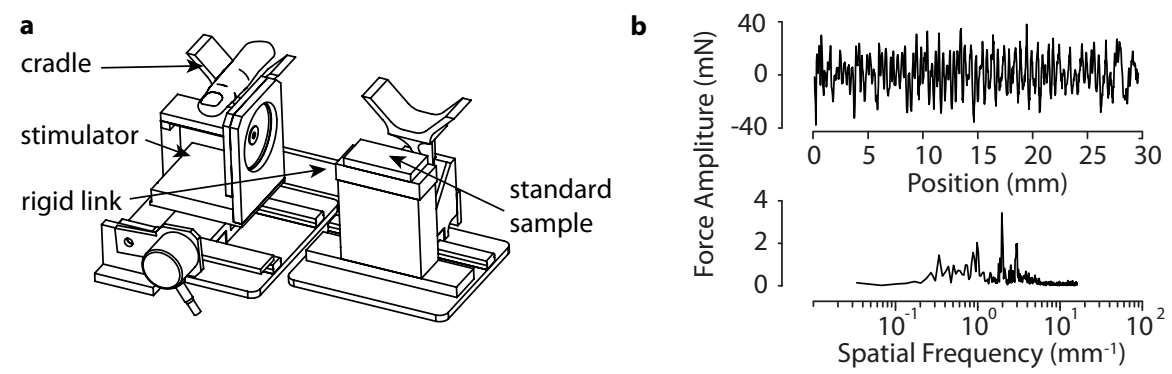

Fig. 4. a: Experimental setup. b: Stimulus and its spectrum. The scanning process transforms a simple surface waveform into a complex, broadband force signal. 
Subjects and Procedure. Six volunteers participated in the experiment, two female and four male, all right-handed, aged from 24 to 31 years. They were from CEA LIST and two of them were familiar with haptic technology. Their hand was guided to explore the setup and after short instructions they were asked to judge whether the standard or the comparison stimulus was rougher and to give an answer via a keystroke. Like in [11], no definition of roughness was given except that "roughness is the opposite of smoothness". Neither training nor feedback was provided during the tests. Gain $G_{\psi}$ was randomly chosen in a range of 1 to 10 . Each value was tested at least 10 times.

\section{Results}

Subjects responded to gain changes following a typical psychometric curve, as shown in Fig. 5. The data were fitted with a cumulative Gaussian distribution $f(x)=0.5$ $[1+\operatorname{erf}((x-\mu) / \sqrt{2} \sigma)]$ where $x$ is the gain, $\mu$ the mean gain and $\sigma^{2}$ the variance. The data fitting was achieved using a nonlinear least-square fitting procedure.

The point of subjective equivalence (PSE) was extracted from the gain that corresponds to a 50\% probability of judging the comparison rougher than the standard. Figure 6 shows the distribution of PSE's. The average across subjects is $\bar{G}_{\psi}=3.99$ with a standard deviation of 0.93 .
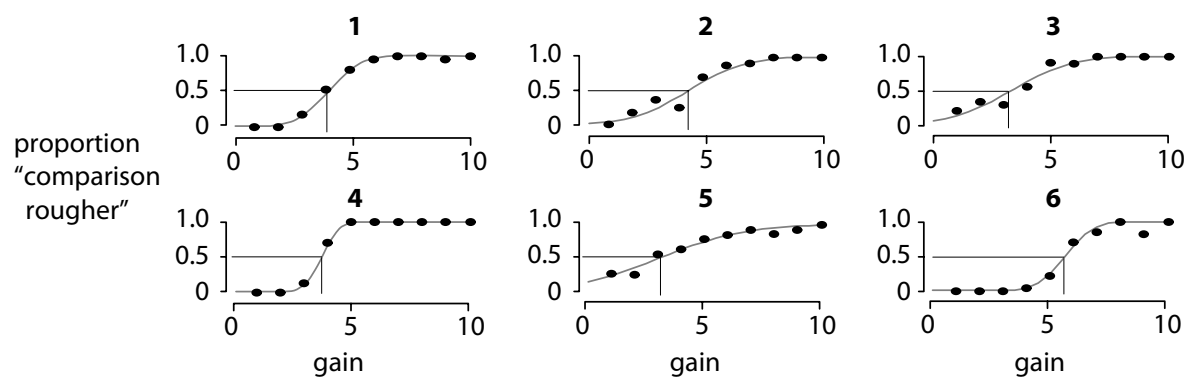

Fig. 5. Results of each participants and their sigmoid fitting

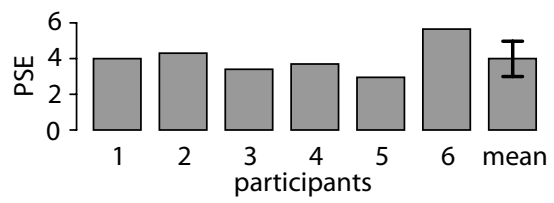

Fig. 6. Value of the Point of Subjective Equivalence of each subject

\section{Discussion}

The results indicate that the interaction force variation can be converted to skin displacement variations to elicit an equivalent sensation of roughness for a virtual surface compared to a real one. As a result, this particularly simple stimulation method is shown to 
be effective at simulating the roughness of a surface. Moreover, participants frequently commented on the perceptual similarity of the sensations themselves between real and simulated surfaces.

These results support the idea that as far as fine textures are concerned, spatial information can be completely eliminated from the simulation, yet, the conscious experience can be that of a non-smooth surface. While similar observations have frequently be reported in the past, our experiments, given to the care that we put in controlling the causality as well as the quality of the transmitted signals, make it now possible to quantify the conditions under which such phenomenon occur. Another aspect of our results worthy of some comments is the relative constancy of the conversion factor among individuals. Of the six individuals who lent themselves to the experiment, five obtained very similar numbers. Only one required a significantly higher displacement stimulus to achieve an equivalent level of roughness. Our efforts will be directed in the future at understanding these individual differences.

\section{Conclusion}

With the help of a carefully engineered sensor, sliding frictional forces could be acquired within a very high dynamic range. The same device was turned in a stimulator having, by construction, a compatible dynamic range that could convert this frictional force into a displacement able to provide a simulated sensation.

This study has so-far considered only one texture for perceptual calibration. We plan to investigate other aspects of texture signals, such as spectral content, in addition to amplitude, and to study the conditions under which perceptual equivalence can be achieved. The distribution of roughness perception across gain values was found to be a monotonic function. As a result, one could employ fast calibration procedures such as accelerated staircase methods as in [11].

A final implication of the present experiment is the possibility to replace force feedback stimulation by cutaneous displacement stimulators which may lend themselves to more favorable engineerings tradeoffs, particularly with subminiature devices. Such miniature devices could for instance be embedded in the gripping surfaces of conventional force feedback devices.

Acknowledgment. The authors would like to thanks Margarita Anastanova for her helpful comments on the experimental setup. This work was supported by the French research agency through the REACTIVE project (ANR-07-TECSAN-020).

\section{References}

1. Lederman, S.J., Taylor, M.M.: Fingertip force, surface geometry, and the perception of roughness by active touch. Perception \& Psychophysics 12(5), 401-408 (1972)

2. Campion, G., Hayward, V.: On the synthesis of haptic textures. IEEE Transactions on Robotics 24(3), 527-536 (2008)

3. Yamamoto, A., Nagasawa, S., Yamamoto, H., Higuchi, T.: Electrostatic tactile display with thin film slider and its application to tactile telepresentation systems. IEEE Transactions on Visualization and Computer Graphics 12(2), 168-177 (2006) 
4. Takasaki, M., Kotani, H., Mizuno, T., Nara, T.: Transparent surface acoustic wave tactile display. In: IEEE/RSJ International Conference on Intelligent Robots and Systems, IROS 2005, pp. 3354-3359 (2005)

5. Biet, M., Giraud, F., Lemaire-Semail, B.: Squeeze film effect for the design of an ultrasonic tactile plate. IEEE Transactions on Ultrasonics, Ferroelectrics and Frequency Control 54(12), 2678-2688 (2007)

6. Winfield, L., Glassmire, J., Colgate, J.E., Peshkin, M.: T-PaD: tactile pattern display through variable friction reduction. In: Second Joint EuroHaptics Conference and Symposium on Haptic Interfaces for Virtual Environment and Teleoperator Systems. World Haptics, pp. 421-426 (2007)

7. Smith, A.M., Chapman, E.C., Deslandes, M., Langlais, J.S., Thibodeau, M.P.: Role of friction and tangential force variation in the subjective scaling of tactile roughness. Experimental Brain Research 144(2), 211-223 (2002)

8. Smith, A.M., Basile, G., Theriault-Groom, J., Fortier-Poisson, P., Campion, G., Hayward, V.: Roughness of simulated surfaces examined with a haptic tool; effects of spatial period, friction, and resistance amplitude. Experimental Brain Research 202(1), 33-43 (2010)

9. Maeno, T., Otokawa, K., Konyo, M.: Tactile display of surface texture by use of amplitude modulation of ultrasonic vibration. In: IEEE Ultrasonics Symposium, pp. 62-65 (2006)

10. Hogan, N.: Impedance control: An approach to manipulation. Journal of Dynamic Systems, Measurements, and Control 107, 1-7 (1985)

11. Campion, G., Hayward, V.: Fast calibration of haptic texture synthesis algorithms. IEEE Transactions on Haptics 2(2), 85-93 (2009) 